\title{
Efeito do extrato de Ginkgo biloba L., Ginkgoaceae, na osteoporose induzida em ratas Wistar
}

\author{
Leda M. F. Lucinda, ${ }^{* 1}$ Beatriz J. Vieira, ${ }^{1}$ Pollyanna A. Salvador, ${ }^{1}$ Tânia T. de Oliveira, ${ }^{2}$ \\ Vera M. Peters, ${ }^{1}$ João E. de P. Reis, ${ }^{1}$ Martha de O. Guerra ${ }^{1}$
}

\author{
${ }^{1}$ Centro de Biologia da Reprodução, Universidade Federal de Juiz de Fora, Caixa Postal 328, 36001-970 \\ Juiz de Fora-MG, Brasil, \\ ${ }^{2}$ Departamento de Bioquímica e Biologia Molecular, Universidade Federal de Viçosa, Vila Gianetti, casa 26, \\ 36570-000 Viçosa-MG, Brasil.
}

\begin{abstract}
RESUMO: Este trabalho investigou os efeitos do tratamento por vinte dias com extrato de Ginkgo biloba (EGb) na osteoporose induzida por glicocorticóides. Foram utilizadas 36 ratas divididas em seis grupos ( $\mathrm{n}=6$ ): Controle, osteoporose, controle positivo, EGb1 (14 mg EGb/mg/kg/dia), EGb2 (28 mg EGb/kg/dia) e EGb3 (56 mg EGb/kg/dia). Os tratamentos foram realizados por vinte dias, após a indução da osteoporose. Após a eutanásia foram removidos o fêmur e a mandíbula de todos os animais. A mandíbula esquerda foi radiografada digitalmente para avaliação da cortical e do suporte ósseo periodontal (SOP). A análise histomorfométrica foi realizada no fêmur e mandíbula direitos. O grupo controle foi comparado ao grupo osteoporose (Teste t de Student) e os demais grupos foram submetidos a ANOVA, seguido do teste post-hoc de Dunnett. Houve redução significava do SOP mesial, percentual ósseo alveolar (POA) mandibular, percentual ósseo trabecular (POT) do fêmur no grupo osteoporose. Houve aumento do SOP mesial no grupo controle positivo, EGb2 e EGb3. O POA da mandíbula e o POT do fêmur aumentaram nos grupos EGb2 e EGb3. O EGb nas doses de $28 \mathrm{mg} / \mathrm{kg}$ e $56 \mathrm{mg} / \mathrm{kg}$ recuperou de forma significativa o SOP mesial, o POA da mandíbula e o POT do fêmur.
\end{abstract}

Unitermos: Ginkgo biloba, osteoporose, radiografia, histomorfometria, alendronato de sódio, ratos.

\begin{abstract}
Effect of the aqueous extract of Ginkgo biloba L., Ginkgoaceae, in induced osteoporosis in Wistar rats.” The objective of this study was to investigate the effect of a 20 day treatment with extract of Ginkgo biloba (EGb) in glucocorticoid-induced-osteoporosis. 36 rats were divided into six groups ( $\mathrm{n}=6$ ): control, osteoporosis, positive control, EGb1 (14 mg EGb/kg/day), EGb2 (28 mg EGb/kg/day) and EGb3 (56 mg EGb/kg/day). Treatments were conducted for twenty days, after osteoporosis was induced. Following euthanasia the femur and mandible of all animals were removed. The left mandible was radiographed to evaluate the cortical and the periodontal bone support (PBS). The histomorphometric analysis was performed on the right mandible and the right femur. The control group was compared with the osteoporosis group (Student's t-test). The other groups were analyzed through ANOVA test followed by Dunnett post-hoc test. There was a significantly reduction in the mesial PBS, in the percentage of the alveolar bone (PAB) of the mandible and percentage of the trabecular bone (PTB) of the femur in the osteoporosis group. There was an increase in the mesial PBS in the positive control group, EGb2 and EGb3. The PAB of the mandible and the PTB of the femur increased in the EGb2 and EGb3 groups. The EGb in the $28 \mathrm{mg} / \mathrm{kg}$ and $56 \mathrm{mg} / \mathrm{kg}$ doses were effective to increase the mesial PBS, the PAB of the mandible and the PTB of the femur.
\end{abstract}

Keywords: Ginkgo biloba, osteoporosis, radiography, histomorphometric, sodium alendronate, rats.

\section{INTRODUÇÃO}

A osteoporose é caracterizada por uma redução na quantidade de tecido ósseo por unidade de volume (Hallworth, 1998) e por uma deteriorização da microarquitetura deste tecido. Essas alterações têm grande impacto tanto na medicina quanto na odontologia, uma vez que a última, necessita da manutenção da quantidade e qualidade de tecido ósseo, para que haja sucesso no tratamento, realizado por algumas especialidades como 
a cirurgia, implantodontia, periodontia, ortodontia e prótese.

Além das complicações na região maxillo-facial a osteoporose causa fraturas que afetam a qualidade de vida principalmente de indivíduos idosos e mulheres na pósmenopausa (WHO, 2003). Somente no Brasil esta doença afeta mais de 10 milhões de pessoas (Rodrigues, 2004). Entre as principais causas da osteoporose encontramse: queda dos níveis hormonais de estrogênio e o uso de alguns medicamentos, como os glicocorticóides, nesse último caso sendo denominada osteoporose secundária.

Os glicocorticóides são antiinflamatórios esteroidais amplamente utilizados no tratamento de doenças auto-imunes, asma, doenças do colágeno, doença renal crônica, entre outras Um dos efeitos colaterais importantes é o impacto negativo sobre a massa óssea. Fraturas ocorrem em $30-50 \%$ dos pacientes expostos a excessos de glicocorticóides (Shaker \& Lukert, 2005).

Os bifosfonatos são drogas muito eficientes no tratamento da osteoporose, porém alguns efeitos adversos destes medicamentos têm sido relatados, tais como: intolerância gastrointestinal (Szejnfeld, 2000) e oteonecrose mandibular (Pozzi et al., 2007). Dessa forma é interessante a busca por alternativas ao tratamento da osteoporose. Dentre essas alternativas estão os fitoestrógenos que têm apresentado benefícios na prevenção e tratamento de doenças cardiovasculares, osteoporose, diabetes e obesidade, sintomas da menopausa, doença renal e vários tipos de câncer (Dang \& Lowic, 2005; Das et al., 2005).

O extrato padrão (EGb 761) de Ginkgo biloba possui $24 \%$ de fitoestrógenos em sua composição, sendo os principais o kaempferol, quercetina e ishorhamnetina (Oh \& Chung, 2004). Destes componentes a quercetina e o kaempferol foram efetivos em inibir in vitro a atividade de reabsorção dos osteoclastos (Wattel et al., 2003). Além dos fitoestrógenos, o extrato de Ginkgo biloba (EGb) possui em sua composição polifenóis antioxidativos, que podem prevenir a perda óssea e o risco de fraturas (Brayboy et al., 2001).

O EGb, in vitro, causou aumento significativo de fosfatase alcalina óssea, promovendo mineralização pelos osteoblastos (Oh et al., 2008). Estudo recente com administração oral do EGb 761 demonstrou aumento da massa óssea de ratas com osteoporose induzida por ovariectomia (Trivedi et al., 2009).

Diante do exposto e na busca de tratamentos novos e alternativos para a osteoporose, este trabalho se propõe a avaliar os efeitos do EGb sobre a osteoporose mandibular e femural, induzida por glicocorticóides, em ratas Wistar.

\section{MATERIAIS E MÉTODOS}

A metodologia deste trabalho foi aprovada pelo comitê de ética em experimentação animal (protocolo número 58/2006-CEEA, Universidade Federal de Juiz de
Fora, MG, Brasil), que segue os princípios internacionais de ética na experimentação animal.

\section{Extrato de Ginkgo biloba}

O extrato padrão (761) de Ginkgo Biloba foi adquirido da China (lote no/ 20060818) pela empresa JR FARMA. O teste de qualidade realizado pelo laboratório Galena mostrou que o EGb tinha em sua composição química: $28,2 \%$ de Ginkgoflavanoglicosídeos, 8,3\% de terpenolactonas, $15 \%$ de glicosídeos quercetina, 10,9\% de glicosídeos kaempferol, 2,3\% de glicosídeos isorhamenitina e menos de 5 ppm de ácidos ginkgólicos. A diluição do EGb761 foi preparada de modo a conter em $1 \mathrm{~mL}$ de água destilada a dose do fototerápico a ser administrado a cada animal por dia (Pinto et al., 2007).

\section{Alendronato de sódio}

A solução de alendronato de sódio foi fornecida pela JR FARMA (Lote da solução no/20060501).

\section{Animais}

Foram utilizadas 36 ratas Wistar, com 50 dias de idade, pesando entre 100-150 g, obtidas na colônia do Biotério do Centro de Biologia da Reprodução, da Universidade Federal de Juiz de Fora. Todos os animais foram alojados em gaiolas de polipropileno, cobertas com camas de maravalha selecionadas (não esterilizada), dotadas de cocho para ração do tipo peletizada e local para mamadeira com água filtrada. Cada gaiola continha três animais, que foram mantidos em armários climatizados (Alesco), localizados em alojamento com regime de luz de 12 h claro/12 h escuro, controlado automaticamente. Os animais receberam uma média de 25 g de ração por dia e água ad libitum (Nepomuceno et al., 2005).

\section{Indução de osteoporose}

A indução de osteoporose foi realizada em todos os grupos, exceto o controle, através da injeção de fosfato dissódico de dexametasona (Decadron ${ }^{\circledR}$ - $4 \mathrm{mg} / \mathrm{mL}$ ) por via intramuscular, na dose de $7 \mathrm{mg} / \mathrm{kg}$ de peso corporal, uma vez por semana, durante cinco semanas (Pinto et al., 2006).

\section{Grupos experimentais}

Os 36 animais foram distribuídos aleatoriamente em seis grupos de seis ratas cada, compreendendo: 1. osteoporose (dexametasona); 2. controle positivo (alendronato de sódio $0,2 \mathrm{mg} / \mathrm{kg} / \mathrm{animal} / \mathrm{dia}$, administrado por via intragástrica, uma vez ao dia, durante vinte dias após a indução de osteoporose) (Pinto et al., 2006); 3. EGb1(Ginkgo biloba $14 \mathrm{mg} / \mathrm{kg} / \mathrm{dia}$ ); 4. EGb2 (Ginkgo 
biloba 28 mg/kg/dia); 5. EGb3 (Ginkgo biloba 56 mg/kg/ dia); 6. Controle (não submetido a qualquer tratamento).

O EGb foi administrado, após a indução de osteoporose, diariamente por via intragástrica durante vinte dias. A escolha da dose de EGb foi baseada em estudo anterior (Pinto et al., 2007), que sugeriu um efeito estrogênico do EGb na dose de $14 \mathrm{mg} / \mathrm{kg} / \mathrm{dia}$ em ratas prenhes. As demais doses foram múltiplas da primeira.

Após 20 dias de tratamento os animais de todos os grupos foram eutanasiados por excesso de anestesia (180 mg/kg, ketamina e $10 \mathrm{mg} / \mathrm{kg}$, xilazina por via intraperitoneal). Após a eutanásia a mandíbula e o fêmur direito de cada animal foram removidos e fixados em formol tamponado a $10 \%$ durante $72 \mathrm{~h}$.

\section{Análise radiográfica}

A mandíbula foi desarticulada, e a mandíbula esquerda foi radiografada, utilizando aparelho de raios-X (Dabi Atlante modelo Spectro 70X, classe I, tipo B comum) com $70 \mathrm{Kvp}, 7 \mathrm{~mA}$ e o sistema de imagem radiográfica intra-oral digital (Computer Dental Radiography-Schick Technologies, Long Island, NY, USA) para análise da espessura da cortical mandibular e do suporte ósseo periodontal (Souza, 2005; Yang et al.,2005).

A mandíbula foi posicionada com o lado lingual sobre a face ativa do sensor digital de maneira que as faces proximais dos molares ficassem paralelas ao feixe central de radiação, que incidiu perpendicularmente ao sensor. As imagens foram obtidas com uma distância focal padronizada de $40 \mathrm{~cm}$ e tempo de exposição de 0,1 s. As imagens foram editadas através do programa Schick Technologies, e foi incluída a elas uma escala de cinza para facilitar a identificação dos pontos a serem analisados. As medidas radiográficas foram realizadas por um único avaliador, que não tinha conhecimento dos grupos experimentais.

\section{Análise da espessura cortical mandibular}

A cortical foi medida em três localizações de sua borda inferior; na região anterior, logo abaixo do ponto de emergência do incisivo mandibular, no ponto mais posterior no ângulo da mandíbula e no terceiro ponto que se localizava no ponto médio entre os dois ( $\mathrm{A}, \mathrm{B}$ e C) (Yang et al., 2005). As mensurações foram realizadas através do software Auto Cad 2002. O valor médio da espessura cortical de cada mandíbula foi obtido dessas três mensurações.

\section{Análise do suporte ósseo periodontal (SOP)}

As mensurações foram realizadas através do software Auto Cad 2002 na raiz distal e mesial do primeiro molar. Nas radiografias digitais foram considerados três pontos: Ápice da raiz distal/mesial (D), crista óssea na superfície distal/mesial do dente (E) e ponta da cúspide distal/mesial (F). Foram mensuradas as distâncias lineares entre os pontos DE e DF, para cálculo do SOP junto à raiz, por meio da fórmula matemática: DE/DF x 100 (Souza, 2005).

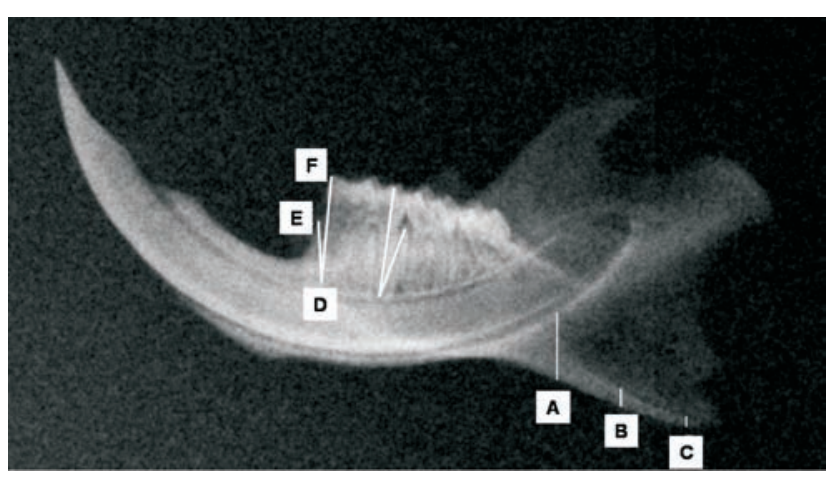

Figura 1. Medida da cortical mandibular, onde A foi o ponto mais anterior na cortical, $\mathrm{C}$ o ponto mais posterior e B o ponto médio entre eles. Medida do suporte ósseo periodontal, onde D corresponde ao ápice radicular, $\mathrm{E}$ à crista óssea e $\mathrm{F}$ à ponta da cúspide do primeiro molar.

\section{Análise histomorfométrica}

Após fixação, a mandíbula direita e o fêmur direito foram descalcificados em solução de ácido fórmico/citrato de sódio por uma semana e incluídos em parafina. Foram obtidos cortes frontais da mandíbula e cortes longitudinais do fêmur de $5 \mu \mathrm{m}$ de espessura em micrótomo histológico rotativo. Logo após, os cortes foram montados sobre lâmina de vidro e corados com hematoxilina e eosina.

As amostras da mandíbula e do fêmur foram analisadas no microscópico óptico Zeiss (Hallbergmoos, Germany) para captura digital de imagens.

\section{Análise histomorfométrica do fêmur}

A região de tecido ósseo trabecular da epífise proximal do fêmur foi fotografada com aumento de $25 x$, determinando-se visualmente três áreas, duas laterais e uma medial (A,B e C), como demonstrado na Figura 2A. Cada uma dessas regiões (A,B e C) tinham a medida de sua área, correspondente a $16000 \mu^{2}$.

Através de uma ferramenta auxiliar presente no software Axiovision ${ }^{\circledR}$ (version 4.5 for Windows semiautomático) utilizado neste estudo, seguiu-se a circundação e a mensuração do tecido ósseo trabecular, com auxílio de uma caneta cursor digital. A área de tecido ósseo obtida foi convertida em proporção de tecido ósseo através da formula \% de tecido ósseo = área obtida na mensuração x 100/16000 $\mu^{2}$. Após serem estabelecidas as proporções de tecido ósseo das três regiões, foi feita a média da proporção de A, B e C correspondentes à epífise proximal do fêmur. Essa média foi posteriormente utilizada para o processamento estatístico. 


\section{Análise histomorfométrica da mandíbula}

A análise histomorfométrica do osso alveolar da mandíbula foi realizada na região do septo interradicular do segundo molar. O procedimento para mensuração foi o mesmo utilizado anteriormente no fêmur, exceto que somente a região de tecido ósseo limitada ao septo interradicular foi mensurada (Figura 2B).

\section{Análise estatística}

O grupo controle foi comparado ao grupo osteoporose através do teste $t$ de Student. Os demais grupos, exceto o controle, foram analisado através da análise de variância (ANOVA), seguido por teste post hoc de Dunnett, o valor de $p<0,05$ foi considerado estatisticamente significativo.

\section{RESULTADOS}

$\mathrm{Na}$ análise radiográfica da mandíbula o grupo osteoporose apresentou diminuição estatisticamente significativa do SOP mesial em relação ao grupo controle. O grupo controle positivo apresentou aumento estatisticamente significativo no SOP mesial, assim como o grupo EGb2 e EGb3 quando comparados ao grupo osteoporose. O grupo EGb3 apresentou aumento significativo da espessura da cortical mandibular quando comparado ao grupo osteoporose (Tabela 1).

Na análise histomorfométrica o grupo osteoporose apresentou diminuição estatisticamente significativa do percentual ósseo trabecular (POT) do fêmur e do percentual ósseo alveolar (POA) da mandíbula em relação ao grupo controle. Os grupos EGb2 e EGb3 apresentaram aumento estatisticamente significativo do POT do fêmur e do POA da mandíbula quando foram comparados ao grupo osteoporose.
Tabela 1. Comparação entre o percentual de suporte ósseo periodontal mesial (SOPM) e o suporte ósseo periodontal distal (SOPD) e a espessura cortical mandibular entre os grupos. controle e osteoporose; osteoporose e grupos controle positivo e extrato de Ginkgo biloba.

\begin{tabular}{lccc}
\hline \multicolumn{1}{c}{ Grupos } & SOPM (\%) & SOPD (\%) & $\begin{array}{c}\text { Cortical } \\
(\mathrm{mm})\end{array}$ \\
\hline Controle & $64,66 \pm 6,08$ & $67,00 \pm 3,40$ & $1,69 \pm 0,33$ \\
Osteoporose & $55,33 \pm 1,75^{*}$ & $65,50 \pm 3,61$ & $1,54 \pm 0,28$ \\
Controle positivo & $65,16 \pm 5,98^{* *}$ & $65,00 \pm 5,79$ & $1,74 \pm 0,16$ \\
EGb1 (14 mg/kg) & $55,33 \pm 5,98$ & $66,33 \pm 5,85$ & $1,75 \pm 0,19$ \\
EGb2 (28 mg/kg) & $62,00 \pm 3,84^{* *}$ & $68,16 \pm 3,19$ & $1,82 \pm 0,22$ \\
EGb3 (56 mg/kg) & $63,33 \pm 2,25^{* *}$ & $66,16 \pm 2,13$ & $2,10 \pm 0,27 * *$ \\
\hline
\end{tabular}

Os resultados foram expressos em media \pm desvio padrão. ${ }^{*} p<0.05$ quando comparado ao grupo controle (teste t de Student); $* * p<0.05$ quando os demais grupos, exceto o controle, foram comparados ao grupo osteoporose (teste post hoc de Dunnett).

Tabela 2. Comparação entre o percentual ósseo trabecular (POT) do fêmur e o percentual ósseo alveolar (POA) da mandíbula entre os grupos: controle e osteoporose; osteoporose e grupos controle positivo e extrato de Ginkgo biloba.

\begin{tabular}{lcc}
\hline Grupos & $\begin{array}{c}\text { POT do fêmur } \\
(\%)\end{array}$ & $\begin{array}{c}\text { POA da mandíbula } \\
(\%)\end{array}$ \\
\hline Controle & $37,06 \pm 2,27$ & $61,06 \pm 3,84$ \\
Osteoporose & $21,76 \pm 2,83^{*}$ & $47,67 \pm 1,18^{*}$ \\
Controle positivo & $24,70 \pm 0,39$ & $58,32 \pm 5,86$ \\
EGb1 (14 mg/kg) & $28,44 \pm 3,29$ & $45,97 \pm 1,52$ \\
EGb2 (28 mg/kg) & $34,60 \pm 4,63^{* *}$ & $65,76 \pm 6,21^{* *}$ \\
EGb3 (56 mg/kg) & $33,96 \pm 1,80^{* *}$ & $73,71 \pm 1,91^{* *}$ \\
\hline
\end{tabular}

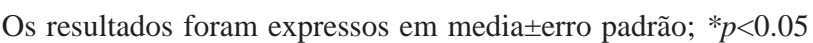
quando comparado ao grupo controle (teste $\mathrm{t}$ de Student); $* * p<0.05$ quando os demais grupos, exceto o controle, foram comparados ao grupo osteoporose (teste post hoc de Dunnett).
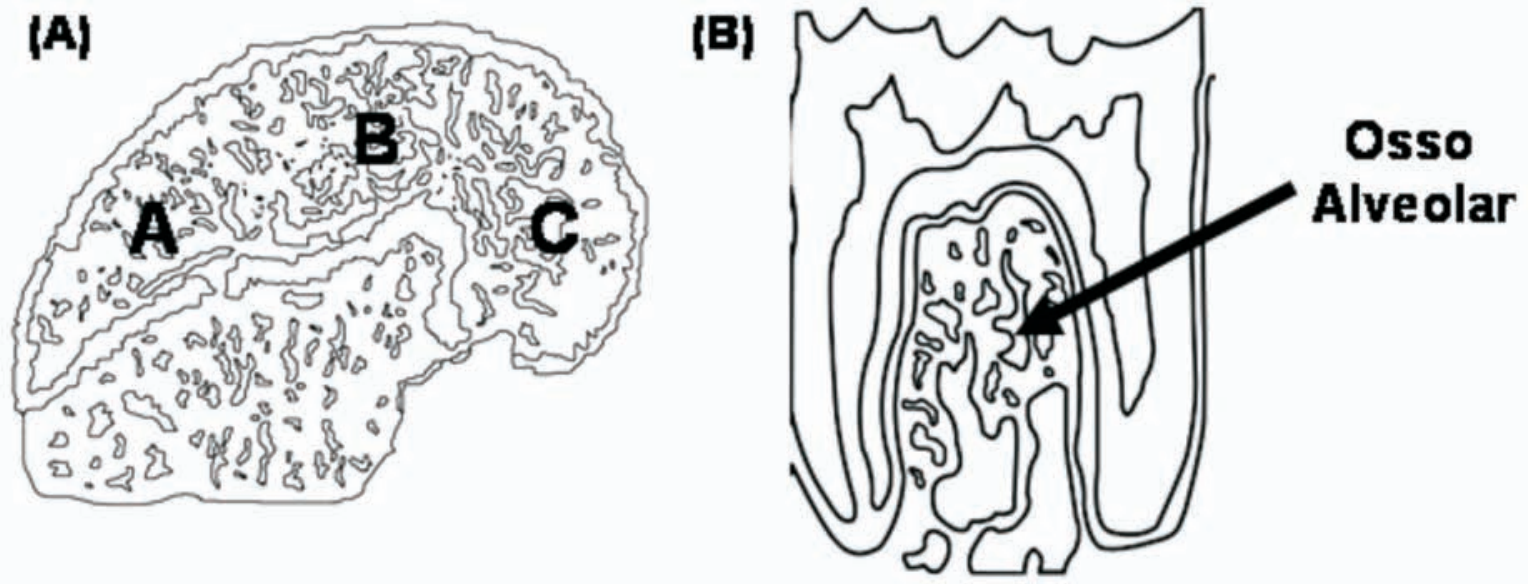

Figura 2. (A) Desenho esquemático da epífise proximal do fêmur, onde $\mathrm{A}, \mathrm{B}$ e $\mathrm{C}$ foram as regiões determinadas para a análise histomorfométrica do tecido ósseo trabecular. (B) Desenho esquemático da região interradicular selecionada para a análise do tecido ósseo alveolar mandibular. 


\section{DISCUSSÃO}

A fisiopatologia da osteoporose induzida por glicocorticóides, ainda não está bem estabelecida, porém a histomorfometria mostra diminuição do volume ósseo total com afilamento das trabéculas ósseas, redução de proliferação e da função dos osteoblastos (Szejnfeld, 2000).

Em estudos clínicos foi verificado que a osteoporose pode levar a um maior comprometimento das estruturas periodontais, diretamente susceptíveis às alterações funcionais e patológicas (Armada et al., 2006), agravando a perda óssea alveolar e aumentando o risco de perda de dentes (Carbonare et al., 2001, Tanaka et al., 2003).

No presente estudo, através da análise radiográfica digital, foi observado que a administração de glicocorticóides causou diminuição significativa do SOPM do primeiro molar mandibular, porém não do SOPD e da cortical mandibular.

Os resultados diferentes entre as regiões estudadas podem ser devidos ao fato, já observado em humanos, de que a alteração óssea mais freqüente na osteoporose ocorre nos ossos que possuem maior percentual de tecido ósseo esponjoso ou trabecular (Byers et al., 1999). No presente estudo a região da crista óssea foi mais atingida devido ao maior teor de tecido ósseo esponjoso, o qual, geralmente, apresenta taxa metabólica maior, respondendo mais rapidamente às mudanças mecânicas do que o osso cortical (Downey \& Siegel, 2006).

Na análisehistomorfométricanotou-sediminuição significativa do POA da mandíbula e do POT do fêmur nos animais tratados com glicocorticóide, evidenciando a redução óssea alveolar mandibular e femural encontrada por outros autores (Fujita et al., 2008; Mahl \& Fontanella, 2008; Pinto et al., 2006; Natsui et al., 2006).

Os dados acima mencionados comprovam a indução da osteoporose pelo glicocorticóide, no modelo experimental usado no presente trabalho.

O alendronato de sódio é amplamente utilizado no tratamento da osteoporose e atua reduzindo a atividade reabsortiva dos osteoclastos através da alteração estrutural dos osteoclastos e de sua morte celular (Hugges et al., 1995). O tratamento dos animais osteoporóticos com alendronato de sódio evidenciou aumento significativo da SOPM, mas na análise histomorfométrica não mostrou aumento significativo do POA da mandíbula e do POT do fêmur.

O resultado pouco significativo com o uso do alendronato pode ser devido ao tempo de administração, uma vez que, nos seres humanos, a supressão da reabsorção óssea ocorre somente após três meses de tratamento diário, e o tratamento realizado no presente estudo foi realizado durante vinte dias. Além disso, os bifosfonatos são drogas altamente hidrofílicas, dessa forma são pobremente absorvidos pelo trato gastrointestinal, via de administração utilizada no presente estudo e sua ação é mais rápida quando o medicamento é administrado por via intravenosa. Por via oral somente $50 \%$ da droga absorvida é retida no esqueleto, o restante é eliminado na urina sem ser reabsorvido (Drake et al., 2008).

As análises radiográficas de mandíbulas dos grupos tratados com EGb (28 e $56 \mathrm{mg} / \mathrm{kg} /$ dia) mostraram aumento significativo do SOPM e, na dose de $56 \mathrm{mg} /$ $\mathrm{kg} / \mathrm{dia}$, também da espessura da cortical mandibular. Histomorfometricamente, as duas doses maiores causaram aumento da POA da mandíbula e do POT do fêmur. Dessa forma, o EGb nas doses e no período de tratamento do presente estudo, apresentou maior recuperação da osteoporose induzida por glicocorticóides do que o alendronato de sódio.

Os resultados do presente trabalho, embora preliminares com o EGb são promissores, uma vez que o alendronato de sódio mesmo tendo sua eficiência comprovada no tratamento da osteoporose, possui alguns efeitos colaterais importantes como a osteonecrose mandibular (Pozzi et al., 2007), o que restringe sua prescrição, principalmente em pacientes que serão submetidos a cirurgias odontológicas.

Os efeitos observados com o tratamento com EGb podem ser devidos a múltiplas atividades no tecido ósseo, causadas por seu efeito estrogênico (Oh \& Chung, 2004), que inibe a reabsorção e promove a osteogênese (Turner et. al., 1994). Além de aumentar os níveis de fosfatase alcalina óssea (Oh et al., 2008; Prouillet et al., 2004).

O kaempferol e a quercetina, constituintes do EGb, estimulam a apoptose de osteoclastos maduros (Wattel et al., 2003), aumentam a densidade mineral óssea trabecular (Trivedi et al., 2009). O kaempferol estimula os osteoblastos induzindo neoformação óssea (Trivedi et al., 2008).

Na osteoporose secundária ao uso de glicocorticóides há redução do volume ósseo por mecanismos ainda não bem conhecidos, porém estudos in vitro sugerem efeitos diretos sobre as células osteoblásticas, reduzindo sua população por mecanismos de indução de apoptose (Weinstein et al., 1998; Silvestini et al., 2000), que pode ter sido recuperada pela ação osteogênica do EGb e seus componentes.

Em síntese, os resultados encontrados sugerem que o uso do extrato de Ginkgo biloba pode ser uma alternativa para o tratamento da osteoporose, particularmente naquelas induzidas por uso prolongado de corticóides.

\section{AGRADECIMENTOS}

Este trabalho foi financiado pela Fundação Mineira de Amparo à Pesquisa (FAPEMIG)-CDS APQ2069-4.04/07 e Redes Mineiras: 2824/05 e 2827/05.

\section{REFERÊNCIAS}

Armada L, Nogueira CRR, Neves UL, Souza PS, Detogne JP, Armada-Dias L, Moreira RM, Nascimento-Saba CC 
2006. Mandible analysis in sex steroid-deficient rats. Oral Dis 12: 181-186.

Brayboy JR, Chen XW, Lee YS, Anderson JJB 2001. The protective effects of Ginkgo biloba extract (EGb 761) against free radical damage osteoblast-like bone cells (MC3T3-E1) and the proliferative effects of EGb 761 on these cells. Nutr Res 21: 1275-1285.

Byers RJ, Denton J, Hoyland JA, Freemont AJ 1999. Differential patterns of altered bone formation in different bone compartiments in estabilished osteoporosis. J Clin Pathol 52: $23-28$

Carbonare LD, Arlot ME, Chavassieux PM, Roux JP, Portero NR, Meunier PJ 2001. Comparison of trabecular bone microarchitecture and remodeling in glucocorticoidinduced and postmenopausal osteoporosis. J Bone Miner Res 16: 97-103.

Dang GZC, Lowik C 2005. Dose-dependent effects of phytoestrogens on bone. Endrocrinol Metab 16: $207-$ 211.

Das AS, Das D, Mukherjee M, Mukherjee S, Mitra C 2005. Phytoestrogenic effects of black tea extract (Camellia sinensis) in an oophorectomized rat (Rattus novergicus) model of osteoporosis. Life Sci 77: 3049-3057.

Downey PA, Siegel MI 2006. Bone biology and clinical implications for osteoporosis. Phys Ther 83: 77-91.

Drake MT, Clarke MD, Khosla S 2008. Biphosphonates: mechanism of action and role in clinical practice. Mayo Clin Proc 83: 1032-1045.

Fujita Y, Konoo T, Maki K 2008. Short-term treatment prevents glucocorticoid-induce bone debility of the mandible in growing rats. Orthod Craniofac Res 11: 187-195

Hallworth RB 1998. Prevention and treatment of postmenopausal osteoporosis. Pharm World Sci 20: 198-205.

Hugges DE, Wright KR, Uy HL, Sasaki A, Yoneda T, Roodman GD, Mundy GR, Boyce BF 1995. Biphosphonates promote apoptosis in murine osteoclasts in vitro and in vivo. J Bone Miner Res 10: 1478-1487.

Mahl CR, Fontanella V 2008. Evaluation by digital radiography of induced changes in the bone density of the female rat mandible. Dentomaxillofac Radiol 37: 438-444.

Nepomuceno F, Las Casas L, Peters VM, Guerra MO 2005. Desenvolvimento embrionário em ratas tratadas com Hiperacum perforatum durante o período de implantação. Rev Bras Farmacogn 15: 224-228.

Natsui K, Tanaka K, Suda M, Yasoda A, Sakuma Y, Ozasa A, Ozaki S, Nakao K 2006. High-dose glucocorticoid treatment induces rapid loss of trabecular bone mineral density and lean body mass. Osteoporos Int 17: 105-108

Oh SM, Chung KH 2004. Estrogenic activities of Ginkgo biloba extracts. Life Sci 74: 1325-1335.

Oh SM, Kim HR, Chung KH 2008. Effects of Ginkgo biloba on in vitro osteoblasts cells and ovariectomized rat osteoclasts cells. Arch Pharm Res 31: 216-224.

Pinto A, Oliveira TT, Del Carlo RJ, Nagem TJ, Fonseca CC, Moraes GHK, Júnior DBF, Cardoso CA 2006. Efeitos de tratamento combinado de alendronato de sódio; atorvastatina cálcica e ipriflavona na osteoporose induzida com dexamatasona em ratas. Rev Bras Cienc Farm 42: 99-107.

Pinto RM, Fernandes ES, Reis JEP, Peters VM, Guerra MO 2007. Intra-uterine growth retardation after prenatal administration of Ginkgo biloba to rats. Reprod Toxicol 23: 480-485.
Pozzi S, Marcheselli R, Sacchi S, Baldini L, Angrilli F, Pennese E, Quarta G, Stelitano C, Caparotti G, Luminari S, Musto P, Natale D, Broglia C, Cuoghi A, Dini D, Di Tonno P, Leonardi G, Pianezze G, Pitini V, Polimeno G, Ponchio L, Masini L, Musso M, Spriano M, Pollastri G 2007. Biphosphonate-associated osteonecrosis of the jaw: a review of 35 cases and an evaluation of its frequence in multiple myeloma patients. Leuk Lynphoma 48: 18521854.

Prouillet C, Mazière J, Mazière $\mathrm{C}$, Wattel A, Brazier $\mathrm{M}$, Kamel S 2004. Stimulatory effect of naturally occurring flavonols quercetin and kaempferol on alkaline phosphatase activity in MG-63 human osteoblasts through ERK and estrogen receptor pathway. Biochem Pharmacol 67: 1307-1313.

Rodrigues E 2004. Osteoporose ameaça 30 milhões de brasileiros. Revista Comciência, disponível em: <http://www.eesc. usp.br/bioeng/resumonoticia.php?codnoticia=70/>, acesso em 9 de janeiro de 2007.

Shaker JL, Lukert BP 2005. Osteoporosis associated with excess glucocorticoids. Endocrinol Metabol Clin North Am 34: 341-356.

Silvestini G, Ballanti P, Patacchiioli FR, Mocetti P, Di Grezia R, Wedard BM, Angelucci L, Bonucci E 2000. Evaluation of apoptosis and the glucocorticoid receptor in the cartilage growth plate and metaphyseal bone cells of rats after high-dose treatment with corticosterone. Bone 26: 33-42.

Souza DM. 2005. Avaliação óssea alveolar de Rattus novergicus por meio dos métodos radiográfico e morfométrico. Cienc Odontol Bras 8: 77-84.

Szejnfeld VL 2000. Manifestações clínicas. Manifestações clínicas, diagnótico e tratamento. In Osteoporose: Diagnóstico e Tratamento. São Paulo: Sarvier, p 353-63.

Tanaka M, Toyooka E, Kohno S, Ozawa H, Ejiri S 2003. Longterm changes in trabecular structure of aged rat alveolar bone after ovariectomy. Oral Surg Oral Med Oral Pathol Oral Radiol Endod 95: 495-502.

Trivedi R, Kumar A, Gupta V, Kumar S, Nagar GK, Romero JR, Dwivedi AK, Chattopadhyay N 2009. Effects of EGb 761 on bone mineral density, bone microstructure, and osteoblast function: Possible roles of quercetin and Kaempferol. Mol Cell Endocrinol 302: 86-91.

Trivedi R, Kumar S, Kumar A, Siddiqui JA, Swarnkar G, Gupta V, Kendurker A, Dwivedi AK, Romero JR, Chattopadhyay N 2008. Kaempferol has osteogenic effect in ovariectomized adult Sprague-Dawley rats. Mol Cell Endocrinol 289: 85-93.

Turner RT, Riggs L, Spelsberg TC 1994. Skeletal effects of estrogen. Endocr Rev 15: 275-299.

Wattel A, Kamel S, Mentaverri R, Lorget F, Prouillet C, Petit JP, Fardelonne P, Brazier M 2003. Potent inhibitory effect of naturally occurring flavonoids quercetin and kaempferol on in vitro osteoclastic bone resorption. Biochem Pharmacol 65: 35-42.

Weinstein RS, Jilka RL, Parfitt AM, Manologas SC 1998. Inhibition of osteoblastogenesis and promotion of osteoblasts and osteocytes by glucocorticoids. J Clin Invest 102: 274-282.

World Health Organization 2003. Prevention and management of osteoporosis. WHO Technical Report Series 921.

Yang J, Farnell D, Devlin H, Horner K, Grahan J 2005. The effect of ovariectomy on mandibular cortical thickness in the rat. J Dent 33: 123-129. 